\title{
(Breve) historia del margen en Julio Ramón Ribeyro
}

\author{
Paul BAUDRY \\ Université de Paris-Sorbonne
}

\begin{abstract}
RESUMEN
Las razones de la marginación del peruano Julio Ramón Ribeyro (1929-1994) por parte del canon del boom latinoamericano son ampliamente conocidas: una preferencia por el cuento en detrimento de la novela, una estética clásica de raigambre francesa y un perfil de escritor discreto. A este respecto, se suele considerar la relación entre esta marginalidad relativa del autor y la marginalidad como tema de escritura dentro de una causalidad reductora que no explota plenamente las implicancias del concepto de margen. Nuestro artículo propone analizar cómo Ribeyro revierte el proceso de su marginación histórica hasta convertirla en una ventaja mediante el cuestionamiento de las zonalidades que rigieron el campo literario hispánico de los años 1960 y 1970.
\end{abstract}

Palabras clave: Julio Ramón Ribeyro, margen, canon, zonalidad, campo literario, boom latinoamericano.

\section{A (brief) history of the margin in Julio Ramón Ribeyro}

\begin{abstract}
Widely known, the reasons for the marginalization of the Peruvian writer Julio Ramón Ribeyro (1929-1994) by the canon of the Latin American boom, can be summarized in three statements: a preference for the short story at the expense of the novel, a classic aesthetic with French roots, and a profile as a discreet writer. In this respect, the relationship between the said relative marginality of the author and marginality as a writing topic itself is usually considered within a state of reductive causality that does not fully exploit the diverse stakes of the concept of margin. The present article aims to analyze the way in which the mentioned author reverses his own marginalization process to turn it into an advantage by means of questioning the "zonalities" that governed the Hispanic literary field during the 1960s and 1970s.
\end{abstract}

Keywords: Julio Ramón Ribeyro, margin, canon, zonality, literary field, Latin american boom.

SUMARIO: 1. La metáfora crítica y el concepto de zonalidad. 2. Bajo la frontera propia: literatura antiépica y tonalidad menor. 3. ¿Marginalidad del escritor y escritura de la marginalidad? 4. Conclusión.

De espaldas a las exigencias del género-éxito de la modernidad, la novela del boom latinoamericano, y sintiendo una afinidad atemporal por los clásicos franceses 
y en particular decimonónicos, se sabe que, históricamente, el peruano Julio Ramón Ribeyro (1929-1994) ha sido excluido del canon latinoamericano. Su reactualización mediática (obras de teatro, homenajes, estatuaria pública, etc.) y su presencia en las antologías e historias literarias recientes, antes invisible, ha sido el fruto de un gesto de militancia crítica por parte de estudiosos hispanoamericanos (Elmore, Ferreira, Ortega) y europeos (Luchting, Valero, Navascués, Esteban, Minardi) que se han esforzado por extraerlo de ese margen ora voluntario ora involuntario para darle cabida en las preocupaciones centrales de lo contemporáneo. Opacado por la figura tutelar de Mario Vargas Llosa y por su timidez proverbial, Ribeyro supo hacer del margen y por consiguiente de la frontera una morada: imaginariamente dentro del Perú pero circunstancialmente fuera, ninguneado por la crítica latinoamericana pero altamente visible en los manuales escolares de su país, cultor de la pureza del cuento pero defensor de la heterodoxia genérica en Prosas apátridas (1975), el autor de La palabra del mudo estuvo confrontado desde siempre a un in-between socioliterario y estético.

Ante este margen/frontera desde donde escribe, la crítica ha reaccionado de dos maneras: primero, considerando la relación entre esta marginalidad relativa del autor y la marginalidad como tema de escritura dentro de una causalidad reductora (escribe sobre los parias porque fue, en mayor o menor medida, un paria literario) y, segundo, considerando de entrada que su posicionamiento fronterizo ha sido un obstáculo para alcanzar cierto reconocimiento (habría acatado pasiva y resignadamente este marginamiento). Nuestro objetivo es discutir estas dos tendencias para repensar su situación fronteriza no como un obstáculo sino como una ventaja, un capital semántico para Ribeyro mediante una (breve) historia del margen.

En efecto, ¿cuál es la rentabilidad literaria del margen? ¿De qué manera Ribeyro revierte el proceso de su marginalización histórica para convertirlo en un punto positivo para la construcción de su obra? Del hecho marginal (es excluido del boom por factores meramente circunstanciales) a la marginalidad como proyecto (renuncia progresivamente al exitismo y a la novolatría), Ribeyro transforma la frontera en una marca de autor, en un "biografema" (Barthes). En ese sentido, nuestra reflexión plantea tres ejes complementarios que articulan, primero, un inventario de las metáforas críticas que han sido utilizadas para explicar-por-laimagen la exclusión de Ribeyro del canon del boom y que revelan los esfuerzos académicos por valorizarlo; segundo, un análisis del giro estético e ideológico que Ribeyro opera tras la toma de conciencia de este relegamiento para convertirlo en el signo de una identidad propia o "escritura diferencial" (Garbatzsky) en la segunda mitad del siglo XX; y tercero, una nueva mirada a la correlación de los dos puntos anteriores con la plasmación ficcional de la marginalidad que remite a la problemática del canon que lo excluyó. 


\section{La metáfora crítica y el concepto de zonalidad}

Si bien la Historia personal del boom (1972) de José Donoso marca el primer hito en la mitografía de los olvidados versus los endiosados por aquel fenómeno comercial de los años 1960 y 1970, resulta interesante reflexionar sobre los argumentos críticos posteriores que intentaron explicar por qué se excluyó del canon a tal o cual autor hoy imprescindible en la medida en que esta indagación implica una epistemología de la frontera. En efecto, tal como lo señala Marcelo Jurisich, "el concepto de canon no se reduce simplemente a una selección de textos y lecturas, sino que además implica [...] un repertorio de modelos a imitar que determina [...] márgenes y [...] fronteras" (Jurisich, 2008), es decir, la naturaleza selectiva y excluyente del corpus del boom implicaba una serie de "valores deseables" que internalizan/aceptan al autor y otra de "valores indeseables" que lo apartan/excluyen de su epicentro, trazando así una línea divisoria que se desplazaba según parámetros comerciales e ideológicos.

Estudiar quiénes estuvieron dentro y quiénes estuvieron fuera -aunque suene un poco esquemático- equivale a revelar la existencia de un entramado axiológico que justifica una doble zonalidad: por un lado, una zonalidad del adentro al adherir a la novela (total si posible) como género-éxito de la modernidad y al aparato mediático de las grandes editoriales hispánicas bajo la batuta de Carmen Balcells y, por otro lado, una zonalidad del afuera al mostrar una indiferencia ostensible hacia el exitismo nacido del liberalismo y serle fiel a una obra propia, quizás anacrónica, aunque haya que pagar el precio del olvido.

A la pregunta de por qué, históricamente, Ribeyro ha sido relegado a una zonalidad del afuera, periférica o marginal, las respuestas de la crítica se han caracterizado por el recurso a la metáfora en detrimento a menudo de explicaciones mucho más factuales ya que el lectorado europeo sufrió un desencuentro 
idiosincrático ${ }^{1}$ con una obra esencialmente derrotista, estilísticamente sobria y de raigambre francesa que no correspondía a su horizonte de espera. Su marcada preferencia por el cuento (casi $80 \%$ de su producción) y la escasa difusión de sus tres novelas hicieron el resto. Además, desde un punto de vista más personal, cabe añadir que Ribeyro carecía del perfil proactivo de un Vargas Llosa o de un de Carlos Fuentes: el llamado "Mudo" estuvo en las antípodas del escritor omnipresente, carismático y rotundamente seductor porque no le interesaba cruzar esa frontera postural y, al ignorarla, cuestionó en silencio la razón de ser de ese contracampo mediático.

Dentro de este contexto, el recurso a la imagen para explicar la zonalidad del afuera en Ribeyro nos parece significativo en la medida en que, argumentativamente, elude un análisis causal y empírico de su exclusión y, retóricamente, privilegia un acercamiento epifánico de este fenómeno donde se apela a la imaginación y a la fantasía del investigador como lector. En efecto, al pasar revista a las imágenes críticas solicitadas entre 1984 y 2009, es de notar que, en su esfuerzo explicativo y a menudo reivindicativo de la originalidad ribeyriana, todas son pensadas desde una relación hacia lo fronterizo, hacia una demarcación normativa -frontera del canon, frontera del género, frontera del exitismo- que Ribeyro respeta, ignora o infringe.

En 1984, en un homenaje titulado "Ribeyro y las sirenas", Mario Vargas Llosa relee la exclusión de Ribeyro como el costo personal y literario de un esencialismo: "En Prosas apátridas [...] el temor al ridículo aleja al escritor contemporáneo de esos grandes temas y lo lleva a confinarse a veces en intrascendencias, sobre las que cualquier idea puede parecer original. Sin proponérselo [...] Ribeyro ha hecho lo contrario: tomar el toro por las astas, ir a lo esencial" (Vargas Llosa, 1996: 262). El concepto de esencia, en tanto naturaleza permanente e invariable, remite, por ejemplo, al objeto de la búsqueda filosófica en la producción ribeyriana donde figuran obras como Prosas apátridas (1975) pero también Dichos de Luder (1989).

1 “Comprendo ahora con mayor claridad que lo que le resta audiencia y repercusión a mi obra literaria es su carácter antiépico, cuando el grueso de los lectores de narrativa anhelan la epopeya. El lector europeo, que vive en un mundo no épico, por simple necesidad de evasión y de contraste busca en la literatura latinoamericana las grandes acciones, los personajes coloreados, los inmensos espacios, las fuerzas telúricas, los fenómenos sociales o de grupo. Todo ellos se encuentra en García Márquez, Asturias, Rulfo, Vargas Llosa, Arguedas, etc. El lector latinoamericano igualmente, pero por razones diferentes (por vivir en un mundo donde es posible la epopeya), busca lo mismo que el lector europeo. Y el mundo de mis libros, hélas, es un mundo más bien sórdido, defectista, donde no ocurre nada grandioso, poblado por pequeños personajes desdichados, sin energía, individualistas y marginados, que viven fuera de la historia, de la naturaleza y de la comunidad" (Ribeyro, 2003: 483). 
Al caracterizarlo como un escritor que torea los circunloquios y apunta hacia formas breves de la sagacidad, Vargas Llosa sitúa a Ribeyro en el paradigma opuesto a la verbosidad de la novela hispanoamericana de los años 1960 y 1970 donde la exhaustividad del acercamiento a la realidad era indisociable de un ideal cuantitativo de palabras. Ir al grano no parece ser una característica de ninguno de los principales novelistas del boom $^{2}$. Parte de esa búsqueda, de ese caminar hacia una verdad o piedra filosofal -Ribeyro era un gran conocedor de la alquimia tanto en su vertiente química como metafísica- se manifiesta en el proceso de depuración de la obra: por ejemplo, de La tentación del fracaso (1971), su diario personal, se extraen fragmentos que integrarán Prosas apátridas (1975) y, a su vez, ésta será la cantera para Dichos de Luder (1989). A la obra total, Ribeyro opone la obra esencial donde la frontera delimita el coto de un arte genuino que hay que proteger, como diría Duras, construyendo un barrage contre le Pacifique.

Prologando la edición de los Cuentos Completos de Alfaguara, en 1994, Alfredo Bryce Echenique caracteriza encomiásticamente a Ribeyro según este mismo paradigma de lo auténtico no solo por los excesos de la amistad sino también porque se inscribe dentro de un esfuerzo colectivo crítico por reivindicar la figura de un olvidado: "Escritos con muchos años de distancia, ['Al pie del acantilado', 'Silvio en el Rosedal' y 'Solo para fumadores'] bastarían para situar a su autor entre los más grandes exponentes de la narrativa breve en el mundo occidental. [...] Su ambición es mayor: ser un arte genuino" (Bryce Echenique, 1996: 124-125). La imagen del artista genuino remite a la construcción de una referencialidad propia que desconoce no tanto la existencia de la norma del canon sino la de su supuesta naturalidad o inmanencia: dicho de otro modo, lo genuino en Ribeyro estriba en su conciencia de la artificialidad de las fronteras del canon en tanto construcciones arbitrarias, convencionales y subjetivas. A Ribeyro no solo no le importa traspasar la frontera comercial trazada por el experimentalismo de las neovanguardias donde brillaron novelas como La casa verde (1966) o Rayuela (1963) sino que se autoinstituye como nuevo centro axiológico, postulando sus preferencias formales por encima de cualquier lobby comercial. En ese sentido, como señala César Ferreira, al escribir una obra "en rebelión silenciosa y en cierta medida a contracorriente" (Ferreira, 2009: 3, la traducción es mía), nuestro autor delimita un perímetro postural alrededor suyo donde la frontera ya no se padece sino que se produce.

${ }^{2}$ La obra y el personaje de Rulfo, anteriores a Ribeyro, comparten esa misma zonalidad del afuera por la heterodoxia de sus perfiles silentes en medio de una cultura del ruido. Rulfo es relegado a la externalidad del "antes" y Ribeyro a la externalidad del "fuera", entre otras razones porque no adhieren a la novela como arte de la digresión que los hubiera conducido a la inautenticidad. 
Esta inversión de los términos que definen su geopolítica literaria ha contribuido en gran medida a que Ribeyro, a defecto de no haber cruzado las puertas del boom, haya entrado en el canon de muchos escritores ${ }^{3}$ por haber tenido la genialidad de hacer de la necesidad una virtud. En efecto, al haber sido relegado voluntaria e involuntariamente hacia una periferia, Ribeyro habita de hecho en una zonalidad marginal donde su propio posicionamiento socioliterario se asemeja curiosamente a la marginalidad como uno de sus temas recurrentes. Las razones de este parecido son múltiples pero la pertinencia del concepto de frontera se manifiesta sobre todo en una de sus consecuencias: la lectura progresista o de izquierda de Ribeyro como autor de gran sensibilidad e incluso compromiso social hacia toda forma de exclusión (social, racial, genérica, etc.). En claro, Ribeyro sería el Dickens peruano por haber vivido en carne propia no la pobreza -aunque sí la indigencia del estudiante parisino en los 1950- sino el ninguneo de un sistema al que, en su juventud, quiso pertenecer.

En 1996, el hispanista francés Roland Forgues lo asocia, en ese sentido, a un "caballero andante del siglo XX": "Si bien es cierto que la aventura de todo gran escritor tiene algo de quijotesco, la de Julio Ramón Ribeyro vivida en el viejo y el nuevo mundo, entre Lima y París donde escribió la mayor parte de su obra, fue probablemente la del último gran caballero andante del siglo XX" (Forgues, 1996: 9). Darle la palabra a los enmudecidos e invisibilizados por la sociedad peruana es una manera de compartir su zonalidad marginal, de ensancharla como espacio horizontal y acogedor, para convertirse, como en el cuento "Por las azoteas" (1958), en el "monarca de las azoteas [que] gobernaba pacíficamente [un] reino de objetos destruidos" (Ribeyro, 2009: 241). Dicho de otro modo, al margen de la Ciudad como los comediantes en Los pájaros de Aristófanes, Ribeyro se convertiría en una figura justiciera que, mediante la representación literaria, critica la fronterización de la sociedad peruana donde una oscura fuerza centrífuga repele a los indeseables, a los que no encajan en su ideal de modernidad literaria o social.

\section{Bajo la frontera propia: literatura antiépica y tonalidad menor}

La identificación de Ribeyro con aquellos que están del otro lado del muro en la periferia conurbana de Lima o en los vericuetos de una clase media empobrecida responde no tanto a una motivación política sino al hecho de que tanto el escritor como sus personajes están ante la misma zanja del éxito como una utopía engañosa.

3 El redescubrimiento de Ribeyro en España, por ejemplo, se realiza mediante la atención que le presta Vila Matas en Bartleby y compañía (2001). Nótese que la fecha es relativamente posterior a su fallecimiento en 1994 y que, a pesar de haber sido publicado por editoriales españolas décadas atrás, Ribeyro recibe solo desde entonces un mejor tratamiento por parte de la crítica peninsular (Navascués, Valero, Esteban, Pérez Esain). 
De este lado, del más acá exitista del boom o del discurso social tecnocrático y progresista de la segunda mitad del siglo XX, reina una actitud que, siguiendo a Meschonnic, podríamos calificar de épica de la novedad. Nuevos rostros, nuevas técnicas, nuevos acercamientos a lo latinoamericano desde la universalidad se multiplican en el centro del boom del mismo modo que, en el centro el poder peruano, se siguen propugnando proyectos modernizadores y que desembocarán, por ejemplo, en la llegada del general Velasco en 1968 y los sueños mesiánicos del primer gobierno revolucionario militar. Para Meschonnic, "la novedad es a la vez real y mítica. Necesita de lo antiguo, puesto que se opone a él. Su surgimiento es glorioso tanto como el prestigio del otro. Y cuando moviliza, es heroico. Esperamos lo nuevo como esperamos a un héroe" (Meschonnic, 1993: 79, la traducción es mía). Así, en Ribeyro, se entiende que la zonalidad del afuera esté regida por la axiología contraria, por lo antiépico, registro y tema que va a adoptar, a transformar en su marca de autor para convertir el margen en un nuevo centro.

¿Pero qué significa una literatura antiépica y por qué toda fronterización del campo socioliterario tiende a producirla? A medida que la ola del boom va avanzado y que autores mucho más jóvenes, como Vargas Llosa, obtienen una atención mediática triplicada por la creación de grandes premios como el Rómulo Gallegos en 1964, Ribeyro va reforzando la representación de sí mismo como paria (literario) hasta consolidar, según Irina Garbatzky, "[una] opción diferencial para la literatura peruana, lejos de lograr la totalidad de las grandes novelas latinoamericanas, [...] 'un tono que se acerca un poco a lo subjetivo, lo arbitrario, lo personal', [esto es,] un tono 'antiépico' [como] contrapartida de la voz 'épica' de [los] narradores consagrados" (Garbatzky, 2009: 128-129). Es decir, Ribeyro da un salto de la marginalidad padecida a la marginalidad asumida en reacción a la épica de la novedad total y a la postura de escritor mediatizado, abriendo así una escritura diferencial que invierte los polos de la frontera.

Esta subversión de las zonalidades será la que la garantice un crepuscular pero renovado éxito a partir de la segunda mitad de los años 1970 y comienzos de los años 1980 cuando el giro postmoderno empiece a desinteresarse por la épica totalizadora del boom y a valorar su figura de escritor escéptico y entregado al fragmento capaz de proponer, según Peter Elmore, un "avanzar más allá de la propia frontera [...], [un] motivo de [...] exploración y [...] búsqueda, pero también de [...] conquista de un nuevo espacio [que prolongue] tanto la subjetividad vertida hacia el diálogo como el escrutinio de sí mismo" (Elmore, 2002: 15). Dicho de otro modo, la fronterización del boom -diferenciar y jerarquizar a los géneros según criterios comerciales- no solo lo incita a hacer del margen un nuevo centro contraponiéndole una escritura antiépica, menor en cuanto a su estridencia mas no sus pretensiones, sino también a transformar su zonalidad del afuera en una zonalidad no del adentro sino de sus adentros.

Este gesto introspectivo, ligado al descubrimiento y al desarrollo de una escritura intimista que busca darle forma a lo informe, desemboca en el 
cuestionamiento, primero, de la geografía de las fronteras genéricas y, segundo, de su supuesta inmanencia. En efecto, tal como lo señala Garbatzky, Ribeyro se adjudica "la tarea [de] realizar una escritura que no someta a las escrituras menores a insertarse dentro de un género mayor. [En] el combate frente al deseo-rechazo del reconocimiento, concluirá afirmando que [...] para que la literatura peruana alcance la literatura universal, deberá extender el campo textual hacia otros géneros" (Garbatzky, 2009: 129). Es decir, tras el rechazo o la indiferencia hacia su obra, Ribeyro no realiza ningún cuestionamiento definitivo de su proceder sino que, al contrario, considera que son los parámetros de medición del canon los que deben ser modificados ensanchando la genericidad literaria o cuestionándola, por ejemplo, con títulos como Prosas apátridas.

La primera edición de 1975 de este primer libro paraficcional provocó un malentendido revelador. Algunos consideraron que eran "las prosas de un apátrida", de un peruano que al cabo de incontables viajes había perdido la noción de su propio origen y donde el marginado sería el propio escritor. Sin embargo, ya en la segunda edición de 1977, Ribeyro añade un paratexto a modo de aclaración: "Se trata, en primer término, de textos que no han encontrado sitio entre mis libros ya publicados y que erraban entre mis papeles, sin destino ni función precisos [...]: no se ajustan cabalmente a ningún género [...]. Carecen de un territorio literario propio" (Ribeyro, 2007: 9). Al no tener capacidad de acción sobre la fronterización del campo literario, Ribeyro se aboca a cuestionar la arbitrariedad de las fronteras genéricas, es decir, lo que funda epistemológicamente la zonalidad del adentro: cada uno de estos fragmentos especulativos, en directa filiación agenérica con $\mathrm{Le}$ spleen de Paris (1869) de Baudelaire, erosiona el muro que lo separa de la oficialidad, de la ortodoxia de los tenores del boom y de las leyes del mercado donde si no se identifica un género, no se vende. Curiosamente, Prosas apátridas marca sin embargo el primer éxito internacional de Ribeyro. ¿Por qué?

\section{3. ¿Marginalidad del escritor y escritura de la marginalidad?}

Sea desde su diario íntimo o desde la ficción misma, la tematización de la exclusión resulta rentable en la medida en que pone de manifiesto aquellas fuerzas socioliterarias y socioeconómicas que relegan al escritor incomprendido y a sus personajes perdedores, respectivamente. Dicho de otro modo, una literatura de la marginalidad es indisociable de una dramatización de los procesos excluyentes $\mathrm{y}$, por consiguiente, tiende a despertar la empatía del lector. En ese sentido, la "apatricidad" de las prosas de Ribeyro (valga el neologismo) suscita la pregunta de según qué o quiénes éstas carecen de patria genérica, situando inmediatamente el texto dentro de un contexto de poder donde las desigualdades entre los capitales de legitimidad -distribuidos oficialmente por el mercado y oficiosamente por los colegas- patentizan las fronteras existentes entre los productores de literatura. 
En Ribeyro, esta revelación de las disimetrías en el campo literario será llevada a la ficción mediante una codificación metafórica de la frontera y de lo fronterizo del mismo modo que, según Pierre Bourdieu y con respecto a Flaubert, se puede leer $L a$ educación sentimental como una puesta en escena de las fronteras ideológicas y estéticas que desgarraban el campo literario donde se movía el novelista francés:

Es posible una lectura $[\ldots]$ que ponga en primer término la estrecha relación existente entre los conflictos sentimentales descritos en dicha novela y las relaciones entre el campo del poder y la situación del artista en tal campo. [En la] correspondencia [de] Flaubert [se] establece [una relación] entre las formas de amor que sucesivamente se le van ofreciendo a Frédéric y las formas de amor por el arte que en la vida bohemia los nuevos artistas están haciendo emerger en ese mismo momento, enfrentando el mundo del arte y el mundo de los negocios, que empiezan a consagrar mecanismos de legitimación muy diferentes, y aun contrapuestos. La ley de incompatibilidad de ambos mundos se cumple a través de la homología entre las formas del amor desinteresado y las formas de amor por el arte que la nueva estética propone: las de un arte no útil, no referencial, autónomo y autosuficiente. (Pozuelo Yvancos y Aradra Sánchez, 2000: 115)

Del mismo modo, en Ribeyro, se puede leer una homología entre la fronterización excluyente del campo literario y la insistencia en las demarcaciones que separan a los artistas - dobles de Ribeyro en la fidelidad a su arte- de los burgueses -dobles de los escritores que gozan de un prestigio adquirido por el boom- Dos (breves) ejemplos.

En el cuento "El embarcadero de la esquina" (1977), Manolo Fuentes organiza un almuerzo de exalumnos de un colegio privado. Los invitados son en su mayoría cincuentones, abogados especialistas en derecho tributario, médicos, arquitectos y ocupan una posición privilegiada en la sociedad limeña. Su clara pertenencia a la burguesía no solo se manifiesta de manera irónica cuando el narrador los califica constantemente como "señores" sino que, ideológicamente, representan un ideario conservador, cartesiano y machista. Son parangones de la oficialidad por oposición a un invitado inesperado: Ángel Devoto, el amigo olvidado, el poeta, el que no se ciñó a un modelo de vida acumulativo. La distancia que lo separa de "los señores" es tanto más grande cuanto que Devoto ha enloquecido, convirtiéndose en un iluminado que profiere galimatías a medio camino entre la absurdidad y la más clara sapiencia.

Al verlo llegar, apestoso y greñudo, los "señores" le ofrecen una copa a cambio de un poema pero Devoto contesta: "Vinazo bueno purita uva respeto para ustedes señores ¿alfombra? Como quieran toda la vida muy chato muy tranquilo o como perro a sus pies así echadito sin ladrar lamiendo sus zapatos" (Ribeyro, 2009: 185). La frontera discursiva entre Devoto y "los señores" remeda la fronterización del campo literario en el que Ribeyro evoluciona. La ausencia de comas entre los sintagmas produce un efecto parataxis que segmenta el discurso encriptándolo del mismo modo que, seguramente, los "señores" o la oficialidad del boom parecieron 
no entender la naturaleza de la poética ribeyriana. Devoto es incomprendido e incomprensible pero, entre líneas, su discurso sibilino está impregnado de sarcasmo: los papeles se invierten y, mediante un recurso quijotesco, el loco es el mejor juez de los cuerdos.

Finalmente, en la obra de teatro Vida y pasión de Santiago el pajarero, reescritura de una de las "tradiciones" de Ricardo Palma, Ribeyro esboza al personaje de Santiago Cárdenas, en la Lima virreinal del siglo XVIII, quien, tras haber observado a los pájaros, pretende inventar un artefacto que le permita volar ${ }^{4}$. Si bien las referencias a Ícaro y Dédalo, así como a la tragedia Los pájaros de Aristófanes, son patentes, en una carta a Wolfgang Luchting citada en 1988, Ribeyro añade la influencia de Brecht, decisiva a la hora de entender esta obra dentro de la frontera que separa (simbólicamente) al artista de la burguesía: "En cuanto a una influencia de Brecht, no tengo muy presente si alguien la ha mencionado. Tal vez a propósito de mi obra de teatro Santiago el pajarero, que es en efecto una especie de versión criolla y diminuta de Galileo Galilei, es decir, una ilustración del choque entre el genio y los poderes temporal y espiritual de su época" (Ribeyro citado en Luchting, 1988: 365). La fronterización del campo socioliterario propio del boom originó ese mismo choque que, en la ficción, opone al idealista Santiago al poder del virrey Manuel de Amat y Junient, y que, en la realidad, opuso históricamente la autenticidad de la poética ribeyriana en tanto obstinación en perseguir un ideal estético sea cual fuere el contexto de recepción y las circunstancias de los "poderes temporales".

Este agôn entre las dos zonalidades -dentro y fuera del canon del boomdesemboca en un enjuiciamiento categórico que, en el seno de la dramaturgia, se simboliza mediante el juicio de Santiago ante la corte por ser culpable de una suerte de crimen de la imaginación:

He dividido mi trabajo en dos partes: la primera versa sobre objeciones teóricas al arte de volar. A la primera objeción teórica la llamo objeción de las alas infinitas. El señor Cárdenas afirma que para que un hombre se sostenga en el aire basta dotarlo de un sistema de alas fabricadas de un material liviano. Estas alas, debido a su gran superficie tendrían por objeto, ofrecer resistencia a la fuerza de la gravedad e impedir la caída del cuerpo volátil. Ahora bien, por livianas que sean, estas alas, tiene un peso, y para que ese peso no origine la caída, será necesario colocar otras alas para las alas. (Ribeyro, 1975: 46)

4 "Con respecto al carácter alusivo de la obra es necesario considerar que el término "volar" posee aquí múltiples connotaciones, que pueden sintetizarse bajo la idea general de "aspirar, estar abierto hacia lo nuevo", aplicable a una actitud del ser humano que supone libertad para practicarla dentro del terreno de las ideas, el arte, la ciencia, el amor, la sociedad, la historia, etc." (Hopkins R., 1984: 137). 
Claramente, el discurso tecnocrático del juez se inscribe dentro de un marco de racionalismo a ultranza que, metafórica y literalmente, pretende hacer bajar a Santiago de su nube. Sin embargo, la parodia de la retórica argumentativa, la multiplicación innecesaria de argumentos, así como el recurso a la tautología ("alas para las alas") remiten a una toma de posición ideológica por parte de Ribeyro que se burla y delezna a los "poderes temporales" que pretenden someter, manipular o anular todo idealismo de raigambre romántica.

\section{Conclusión}

Una (breve) historia del margen en Julio Ramón Ribeyro es entonces indisociable de una profundización de la relación entre marginalidad del escritor y tematización de la marginalidad. Al borde del canon, en la zonalidad del afuera, Ribeyro entiende que puede revertir su supuesto fracaso, estetizarlo en cierta manera (Baudry, 2009: 375-400), y así convertirlo en una marca de autor. Desde la trinchera de aquella tonalidad menor, privilegiando e inaugurando, al menos dentro del canon peruano, el ejercicio de formas breves y reflexivas, Ribeyro crea un contracampo de respuesta a los "poderes temporales" del mercado que habían conseguido invisibilizarlo hasta mediados de los años 1960 y 1970. Si bien la fronterización de los géneros y de los públicos dentro del boom latinoamericano requiere una serie de poderes excluyentes institucionales y editoriales, su debilidad radica en darle a los excluidos, en este caso al excluido, el poder de la revancha, la legitimidad de David contra Goliat, y así, sin quererlo, crearle un lectorado afín a lo antiépico que busque verdades tangenciales, sugeridas, al margen de la autosuficiencia de los centros de poder.

\section{BIBLIOGRAFÍA}

BAUDRY, Paul.

2009 "El pedestal sin estatua. La estetización del fracaso en Dichos d Luder (1989) de Julio Ramón Ribeyro", en Néstor Tenorio Requejo y Jorge Coaguila (ed.), Julio Ramón Ribeyro: penúltimo dossier: homenaje a un clásico de la narrativa hispanoamericana. Trujillo: Tierra Nueva Editores / Universidad Nacional Pedro Ruiz Gallo.

BRYCE ECHENIQUE, Alfredo.

1996 "El arte genuino de Ribeyro" [1994], en Ismael P. Márquez y César Ferreira (eds.), Asedios a Julio Ramón Ribeyro. Lima: Pontificia Universidad Católica del Perú, Fondo Editorial.

ELMORE, Peter.

2002 El perfil de la palabra: la obra de Julio Ramón Ribeyro. Lima: Pontificia Universidad Católica del Perú y Fondo de Cultura Económica, Col. Tierra Firme. 
FERREIRA, César.

2009 "Reading Ribeyro", en Chasqui: peruvian mail, núm.15. Lima: Cultural Bulletin of the Ministry of Foreign Affairs.

FORGUES, Roland.

1996 "Ribeyro, caballero andante del siglo XX", en Néstor Tenorio Requejo (ed.), Julio Ramón Ribeyro: el rumor de la vida. Lima: Arteidea Editores.

GARBATZKY, Irina.

2009 "Las derivas del fracaso. Sobre los diarios de Julio Ramón Ribeyro", CELEHIS - Revista del Centro de Letras Hispanoamericanas, núm. 20, Mar del Plata.

HOPKINS R., Eduardo.

1984 "El teatro de Julio Ramón Ribeyro", en Revista de crítica latinoamericana, año X, núm.20, segundo semestre, Lima.

JURISICH, Marcelo.

2008 "Lo que yace debajo: para qué sirve el canon literario", en Espéculo. Revista de estudios literarios, núm.38, Marzo-Junio, Año XIII, Madrid: Universidad Complutense de Madrid [Consultado el 28/02/13

en:

http://pendientedemigracion.ucm.es/info/especulo/numero38/canonl

MESCHONNIC, Henri. it.html].

1993 Modernité Modernité. Paris: Gallimard, Col. Folio/Essais.

Pozuelo Yvancos, José María, y ARADRA SÁnCHEZ, Rosa María.

2000 Teoría del canon y literatura española. Madrid: Cátedra, Col. Crítica y Estudios Literarios.

RIBEYRO, Julio Ramón.

1975 Santiago el pajarero [1958]. Lima: INC.

1988 Carta a Wolfgang Luchting citada en Wolfgang A. Luchting, Estudiando a Julio Ramón Ribeyro, Iberoamericana / Vervuert: Francfort-sur-le-Main.

2003 La tentación del fracaso, Diario personal (1950-1978) [13/18/76, París]. Barcelona: Seix Barral, Col. Biblioteca Breve.

2007 Prosas apátridas (completas), Barcelona: Seix Barral, col. Biblioteca Breve.

2009 "El embarcadero de la esquina", La palabra del mudo, t. II. Barcelona: Seix Barral.

2009 La palabra del mudo, T. I, Barcelona: Seix Barral.

VARGAS LLOSA, Mario.

1996 "Ribeyro y las sirenas" [1984], en Ismael P. Márquez y César Ferreira (eds.), Asedios a Julio Ramón Ribeyro. Lima: Pontificia Universidad Católica del Perú, Fondo Editorial. 